\title{
Neonatal outcomes and its association among gestational diabetes mellitus with and without depression, anxiety and stress symptoms in Malaysia: A cross-sectional study
}

\begin{abstract}
Objective: Prevalence of depression, anxiety and stress symptoms in gestational diabetes mellitus ranges from $10.2 \%$ to $39.9 \%$ based on previous studies in Malaysia. Presence of depression, anxiety or stress in pregnancy may increase the risk of neonatal morbidity and mortality. The aim of this study was to determine the prevalence of neonatal outcomes and its association among mothers with gestational diabetes mellitus with and without the presence of depression, anxiety and stress symptoms in Malaysia. Design: This was a cross-sectional study. Setting: Tertiary hospitals in Malaysia. Participants: Mothers with gestational diabetes mellitus $(n=418)$ who deliver their neonates at two major tertiary hospitals in Malaysia. Measurements: Neonatal outcomes, such as low birth weight, preterm birth, macrosomia, metabolic and electrolyte disorders, neonatal respiratory distress and congenital anomalies were determined. Findings: Prevalence of low birth weight in neonates born to mothers with gestational diabetes mellitus was $14.6 \%$, followed by metabolic and electrolyte disorders $10.5 \%$, preterm birth 9.1\%, macrosomia 4.8\%, neonatal respiratory distress $5.8 \%$ and congenital anomalies $(2.4 \%)$. Among the adverse neonatal outcomes, neonatal respiratory distress was significantly associated with the presence of depression symptoms in mothers with gestational diabetes mellitus using univariate analysis $(\mathrm{p}=0.010)$. After controlling for confounding factors, predictors for neonatal respiratory distress at delivery were the presence of depression symptoms in mothers with gestational diabetes mellitus (Adjusted OR $=3.87,95 \% \mathrm{CI}=1.32$ 11.35), living without a husband (Adjusted $\mathrm{OR}=9.74,95 \% \mathrm{CI}=2.04-46.51$ ), preterm delivery (Adjusted $\mathrm{OR}=7.20,95 \% \mathrm{CI}=2.23-23.30$ ), caesarean section (Adjusted $\mathrm{OR}=3.33,95 \% \mathrm{CI}$ $=1.09-10.15$ ), being nulliparous and primiparous (Adjusted $\mathrm{OR}=3.62,95 \% \mathrm{CI}=1.17-11.17$ ) and having family history of diabetes (Adjusted $\mathrm{OR}=3.20,95 \% \mathrm{CI}=1.11-9.21$ ). Key conclusions: The findings of this study demonstrate the positive association of neonatal respiratory distress with the presence of depression symptoms in mothers with gestational diabetes mellitus. Implications for practice: It is therefore important to identify depression symptoms after a diagnosis of gestational diabetes mellitus in pregnant mothers is made to enable early referral and interventions.
\end{abstract}

Keyword: Neonate; Morbidity; Depression; Anxiety; Stress; Gestational diabetes 